\title{
Subfascial endoscopic transaxillary breast augmentation: long-term evaluation of 3,004 patients over a 10-year period
}

\author{
Surin Plasen, MD, PhD \\ Praram 3 Cosmetic Surgery Clinic, and Thai Association and Academy of Cosmetic Surgery and Medicine, Bangkok, Thailand
}

\begin{abstract}
Background: Subfascial endoscopic transaxillary breast augmentation were reported the benefits over the submuscular implant since 2000. Long term outcome was not reported in any large number of cases.

Objective: To evaluate the long-term outcome in 3,004 cases over 10-year period.

Methods: From 2007 to 2018, 3,004 cases were operated in our center. 2,949 cases undergo subfascial endoscopic transaxillary breast augmentation with textured round implant, 35 cases with smooth round gel, and 20 cases with textured anatomical implants. Inclusion criteria for patient selection is the pinch test in all patient group and this value is more than 1.5 inch. Post-operative follows up time from 2007 to 2018 with yearly visit with postoperative photograph. Postoperative complications were evaluated by the capsular contracture, abnormal chest muscle movement, rippling, infection, seroma, bottom out, hematoma, implant rotation and implant border palpation.

Results: Early postoperative hematoma was found in $0.16 \%$ in textured round implant and was not found in smooth gel and anatomical textured implant. The rate of capsular contractor was $0.16 \%$ in textured round implant, $20.0 \%$ in smooth gel and $10.0 \%$ in anatomical textured implant. The rate of infection and seroma formation occurs $0.2 \%$ in textured round implant. The rate of rippling is $0.33 \%$ in textured round implant, $10.0 \%$ in anatomical textured implant and no rippling in smooth gel implant.

Conclusion: Subfascial endoscopic transaxillary breast augmentation can be used in patients with thick breast tissues. Rippling and implant border palpation occurs frequently in textured round implant and textured anatomical implant. Capsular contracture is noticeably high in textured anatomical and smooth round implant. Careful selection of the patients is the key to success in this type of breast augmentation.
\end{abstract}

Keywords: breast augmentation; endoscopic; late complication; rippling; sub fascial; textured round implant

\section{Introduction}

Breast augmentation can be accomplished using several incision routes such as periareolar, inframammary, umbilical and axillary incision. Periareolar incision was claimed to be the cause or source of infection due to bacterial contamination of the implant. The use of nipple shields as a routine maneuver to avoid contamination of the implants was proposed [1]. Umbilical incision limits the scar outside the breast up to the umbilicus, but the technical demands require specialized instruments, high skill and a learning curve for the surgeon [2,3]. Inframammary fold incision is selected for revision cases or ptotic breast

Received October 11, 2018; Revised December 6, 2018; Accepted December 7, 2018

Corresponding author: Surin Plasen

E-mail: surinmd9812@gmail.com, ORCID: https://orcid.org/0000-0002-4923-5543

This is an Open Access article distributed under the terms of the Creative Commons Attribution Non-Commercial License (http://creativecommons.org/licenses/by-nc/4.0), which permits unrestricted non-commercial use, distribution, and reproduction in any medium, provided the original work is properly cited.

Copyright @ 2018 Korean Society of Korean Cosmetic Surgery and Medicine (KSKCS \& KCCS). 
and leaves a scar at the base of the breast tissue. Axillary incision is being widely used due to the absence of scars on the breast and the dissection plane align to the anatomical space of the pectoralis major muscle.

Applications of endoscopic magnifying lenses and video amplification of the images improve visual data and enable accurate pocket dissection and hemostasis. Axillary submuscular breast augmentation was performed on 6,972 cases (unpublish data) at our Center between 1987 and 2007. Severe complications of submuscular breast augmentation such as hard capsular contracture with a thick capsule, abnormal breast movement, acute severe hematoma, and poor breast cleavage with lateral movement have been observed. In case of capsular contracture, revision surgery for correction is rather difficult due to very thick capsule formation. Hematoma can occur from the major perforating arteries.

Subfascial endoscopic transaxillary augmentation mammaplasty was initially reported in 2000 [4] and in 2004 [5], and their results were perfect and natural contour. The subfascial route has been our choice for breast augmentation since 2007 . The reasons for this change are as follows: the ability to achieve a perfect breast cleavage that cannot be achieved through submuscular breast augmentation, accurate pocket dissection without the long-term pocket contraction, and no damage to the origin of the pectoralis major muscle. This muscle has important actions for chest and shoulder movements. Subfascial route operation is more minimally invasive than the submuscular route.

\section{Materials and methods}

From April 2007 through May 2018, 3,004 cases were operated at our Center, of which 2,949 cases underwent subfascial endoscopic transaxillary breast augmentation with textured gel (Mentor, siltex implant, moderate profile, cohesive I gel implant), 35 cases with Mentor smooth gel, moderate profile implant and 20 cases with textured anatomical implants (Allergan, Style 410F, Cohesive III). In the first group, 10 cases were from another surgeon, the pockets were changed from a submuscular to a subfascial plane to correct the capsular contracture. The implants were used with sizes ranging from 200 to 600 $\mathrm{ml}$ (average size, $325 \mathrm{ml}$ ). The mean age of the patients was 29 years (ranged from 19 to 61 years). In 20 cases with anatomical implant, the size used ranged from 280 to $450 \mathrm{ml}$ (average size, $295 \mathrm{ml}$ ). In 35 cases where smooth gel implant was used, the size ranged from $250 \mathrm{ml}$ to $600 \mathrm{ml}$ (average size, $350 \mathrm{ml}$ ). The mean patient age for this group was 32 years (ranged from 19 to 59 years) with multiple pregnancies and ptotic breasts. Smooth gel implants were used in small amounts due to the author's prior experience from 1987 to 2007, in that high incidence rates of capsular contracture were observed. The inclusion criteria for patient selection are as follows: the breast tissue thickness by a pinch test should be more than 1.5 inches. If the pinch test indicates less than 1.5 inches thickness, submuscular augmentation will be needed, but is not include in the study protocol.

All the patients were followed up within 3 days, 7 days, 1 month after surgery, and yearly visits, with a recommendation to replace the implant after 10 years of the operation. Hospital admission is not required in all of 3,004 cases.

All the subfascial breast augmentations were performed by 1 surgeon and photographic analyses of all patients were investigated using the standard three-view postoperative photograph by a single surgeon.

The postoperative follow up time from 2007 to 2018, specifically with yearly visits coinciding with the 10-year anniversary of the operation included a special recommendation for replacement of the implant.

Statistical analysis was performed using the number of the incidences observed in each group. The results (postoperative complications) were evaluated based on capsular contracture, abnormal muscle movement, and rippling, which requires a change (of plane) to submuscular plane, infection, seroma, bottom out (descent), and hematoma, which requires emergency surgery, implant rotation, and implant border palpation.

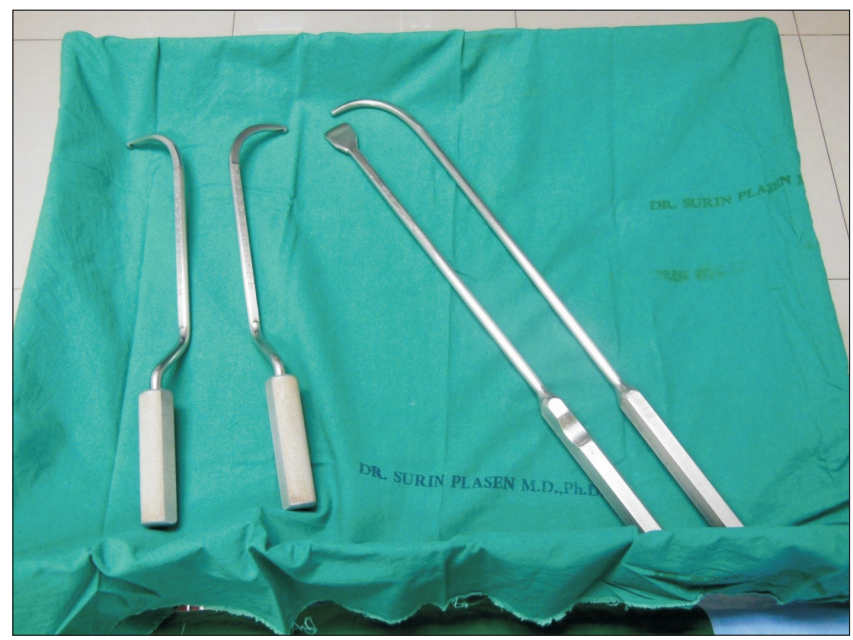

Fig. 1. Special instruments for pocket dissection (right) with long handle for lateral forceful blunt dissection in pocket creation. The instruments on the left is Angrist-Dingman dissector. 


\section{Procedures}

Patients provided informed consent prior to the surgery. Patients were subjected to general anesthesia, with their arms adducted to 90-degrees and head slightly elevated. Ceftriaxone was administered intravenously just prior to surgery, and cephalexin was prescribed for oral intake for 10 days. Approximately 500 to 1,000 ml of Klein's tumescent solution was injected all over the breast tissues and axillary incisions were performed at about 5 to $5.5 \mathrm{~cm}$ in length and $2 \mathrm{~cm}$ posterior to the axillary clavus just below pectoralis major muscle border. With this access, the sub fascial plane could be monitored accurately and performed easily with a special instrument (Fig. 1) all the way up to below the nipple area and upward to the second intercostal space. A $4 \mathrm{~mm}$ endoscope with a special endoretractor was used to bluntly dissect the original inframammary line. Bleeding was cauterized by using electrocautery and endoscopic forceps (Fig. 2). For a larger implant insertion, approximately 1 to $3 \mathrm{~cm}$ dissection of the pocket below the original inframammary fold is required. This dissection requires the use of the endoretractor and endodissector with a longer electrocautery tip as shown in Fig. 2. This is necessary because the adhesion of

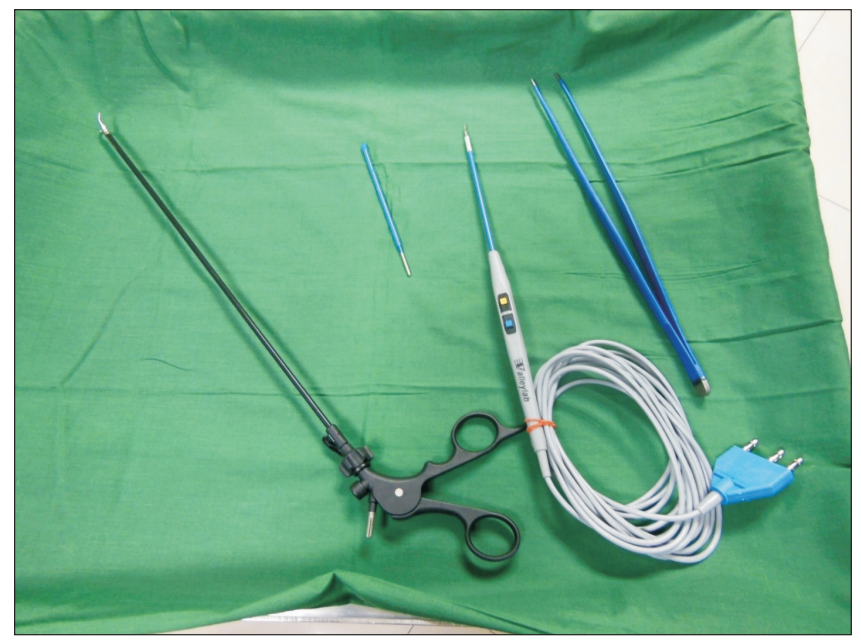

Fig. 2. Electrocautery instruments include long forceps, endoforceps, and long hand switch cautery tip. the muscle fascia such as the serratus anterior, pectoralis major, lateral oblique and rectus abdominis are present in this area $[6,7]$. The perfect subfascial dissection is demonstrated in Fig. 3. The new inframammary fold has been calculated based on the manufacturer's chart for the appropriate implant diameter and projection. Half of the implant diameter plus projection was used to achieve perfect results. No high riding implants were observed. Lateral pocket dissection was done by using a blunt dissection instrument with a long handle (Fig. 1) to reduce the force needed by the dissecting surgeon, and to preserve the fourth intercostal nerve.

The pocket was irrigated with betadine solution prior to inserting the implants at a right angle or with an Army retractor. Bleeding in the pocket was monitored using a fiberoptic retractor. Skin was closed layer by layer with 4/0 Vicryl and 5/0 Ethilon. Drainage was not used in all the cases after rechecking the bleeding points and to prevent bacterial contamination of the pocket and the implant.

\section{Results}

A typical perfect subfascial endoscopic breast augmentation

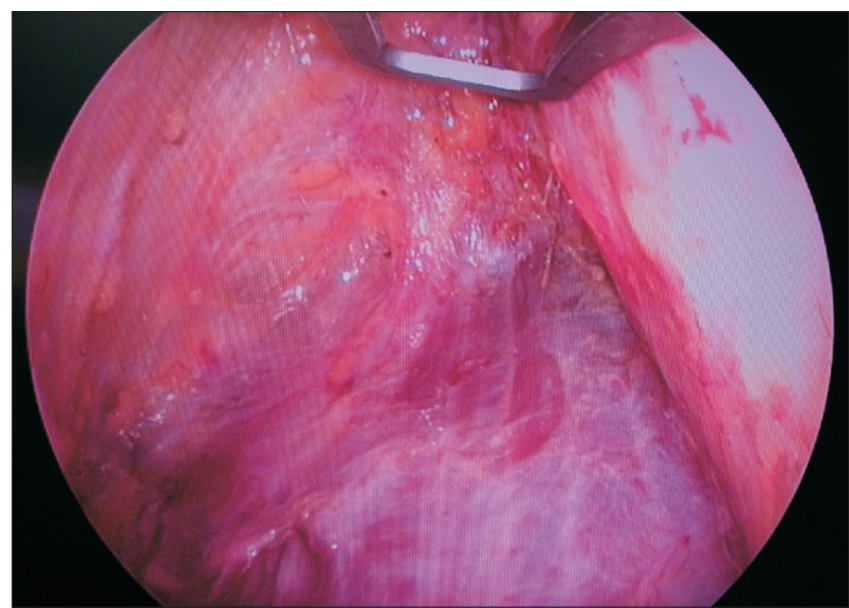

Fig. 3. Perfect subfascial dissection.
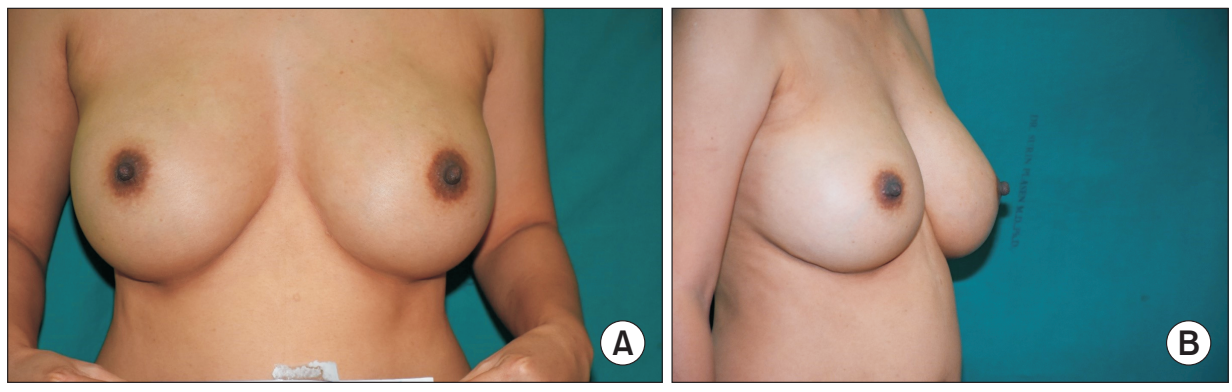

Fig. 4. (A, B) Typical postoperative good subfascial breast augmentation in anterior and oblique view of patient. 
result in a patient is presented in Fig. 4. Table 1 lists the postoperative complications observed. Early post-operative complications in these 3,004 cases include hematoma, which required immediate reoperation, and was observed in $0.16 \%$ ( 5 cases) of patients that underwent subfascial endoscopic transaxillary breast augmentations with textured, round silicone implants. This complication was not observed in the textured anatomical implant group and the smooth gel round implant group.

The differences in the rate of capsular contracture $(20.0 \%$ in smooth gel implant group and $0.16 \%$ in the textured, round moderate profile group) were clearly noticeable. Also, the capsular contracture incidence was high in patients with textured anatomical implant (10.0\%).

Seroma and infection were observed only in textured round implant $(0.2 \%)$, but not in the smooth gel round implant and textured anatomical implant group.

Table 1. Postoperative complications after subfascial endoscopic breast augmentation from 2007 to 2018

\begin{tabular}{lccc}
\hline \multicolumn{1}{c}{ Type of implant } & $\begin{array}{c}\text { Textured } \\
\text { round }\end{array}$ & $\begin{array}{c}\text { Textured } \\
\text { anatomical }\end{array}$ & $\begin{array}{c}\text { Smooth } \\
\text { round }\end{array}$ \\
\hline Patients (n=3,004) & 2,949 & 20 & 35 \\
Years follow up & 10 & 10 & 10 \\
\hline Complication & & & \\
\hline Capsular contracture ${ }^{\text {a) }}$ & $5(0.16)$ & $2(10.0)$ & $7(20.0)$ \\
\hline Muscle movement & - & - & - \\
\hline Rippling & $10(0.33)$ & $2(10.0)$ & - \\
\hline Infection & $3(0.1)$ & - & - \\
\hline Seroma ${ }^{\text {b) }}$ & $3(0.1)$ & - & - \\
\hline Bottom out & $1(0.03)$ & - & - \\
\hline Hematoma & $5(0.16)$ & - & - \\
\hline Implant rotation & - & $2(10.0)$ & - \\
\hline Implant border palpation & $10(0.33)$ & $2(10.0)$ & - \\
\hline
\end{tabular}

Values are presented as number only or number (\%).

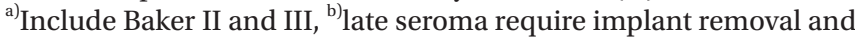
reoperation after 3 months.
One of the specific complications observed in the textured anatomical implant was the implant rotation (10.0\%) requiring revision to correction the problem.

Implant border palpation was observed in both the textured round and textured anatomical implant groups but was not observed with smooth gel implants.

Bottom out (implant descent) was observed in a single case involving a very large textured round implant augmentation ( $450 \mathrm{ml})$.

Further, number of rippling cases, requiring revision surgeries was significantly high in the textured round implant $(0.33 \%)$ and the anatomical textured implant (10.0\%) groups. This type of complication was not observed in the smooth round implant group. Also, rippling was noticed around two-three years after the surgery. The cases in the patient group with disturbing rippling requiring reoperation necessitated changes to the subpectoral pocket. The patient with moderate and severe rippling is shown in Figs. 5 and 6. As mentioned previously, this phenomenon occurs around two-three years after surgery.

\section{Discussion}

Subfascial breast augmentations via axillary approach were

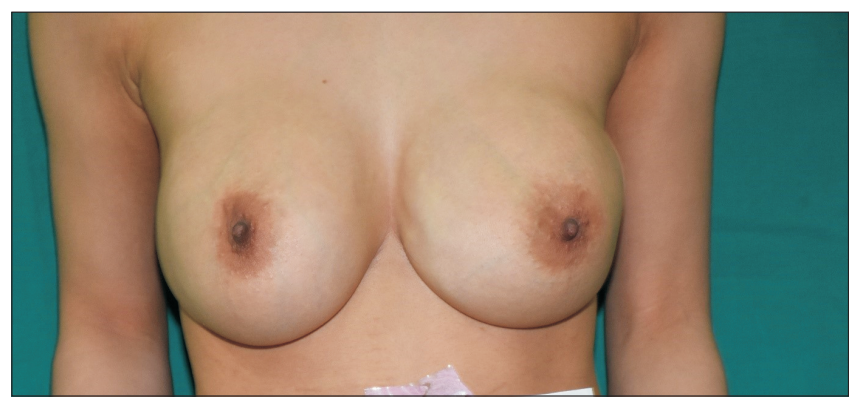

Fig. 5. Typical rippling with soft breast in subfascial breast augmentation after 2 years in 32 - year-old patient.
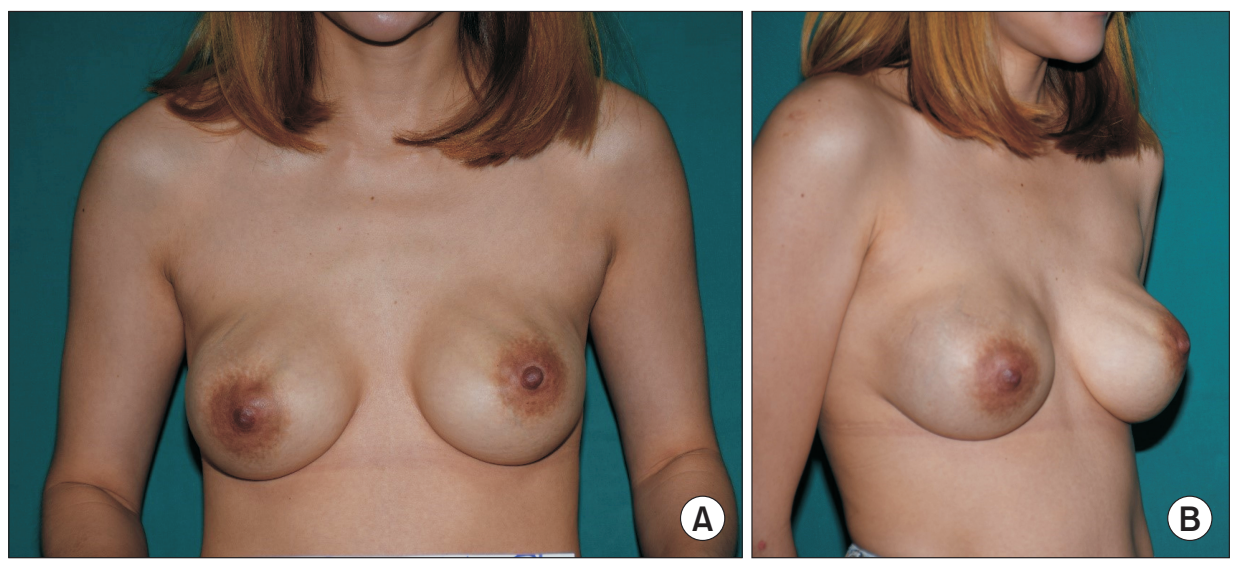

Fig. 6. (A, B) Postoperative view of a 29-year-old patient with severe rippling that requires reoperation to the submuscular plane. 
reported in 2000 [4] and in 2004 [5]. The plane of dissection was changed from submuscular to subfascial plane by these authors. The advantages of the submuscular placement include thick tissues over the implant in the upper part and fewer incidences of superior rippling. The implant edge is, however, not palpable in the upper part of the breast. Also, several disadvantages of submuscular placement exist, which include high invasiveness of surgery requiring the release of the pectoralis major origin, severe post-operative pain, and abnormal implant movement from the contraction of the pectoralis major muscle. There are no definite long-term reports of abnormal chest and shoulder movements. Capsular contracture may occur in submuscular placement and its correction could be very difficult due to the thick capsule and highly vascularized tissues. Postoperative complications including occurrence of hematoma and recurrence of capsular contracture are possible.

The subfascial approach for augmentation mammaplasty was chosen based on the benefits of the technique, such as being a minimally invasive surgery, and little post-operative pain. Also, this technique does not damage the pectoralis major origin. The immediate and long term results were excellent with a good breast cleavage and good breast shape after the operation. The breast cleavage is still one of the most common criteria that the patients seek from the surgery.

The most concerning long-term complication is rippling. In patients with thin breast tissues (pinch test less than 1.5 inches) and in multiparous women, high incidences of rippling without capsular contracture could occur and may require revision surgery to change the plane to a submuscular space. In our experience involving 3,004 cases, the incidences of hematoma, seroma, and infection were not very different from those observed in submuscular placement. Many patients request a good breast cleavage as their priority, so choosing subfascial plane may address this problem. As the incidences of rippling are high, it is suggested to limit this technique for the patients with thick breast tissue. Also, implant border palpation was observed in 10 cases $(0.33 \%)$. This incidence was also found in a patient with thin breast tissue.

The capsular contracture rate is very low in these several subfascial mammaplasty procedures. However, correction of capsular contracture is also easier than with submuscular placement since only a thin capsule develops after subfascial mammaplasty.

The benefit of endoscopy in axillary subfascial augmentation is well known. It basically makes everything visible and transforms a blind surgery to somewhat of an open surgery. The dissection of the pocket and cauterization of the bleeding point is also easily accomplished in this procedure. The subfascial pocket cannot be bluntly dissected closer and downward to the inframammary fold $[6,7]$. As a result, it becomes a surgical plane and not an anatomical plane, and necessitates a sharp dissection to dissect the rectus abdominis fascia in continuity with the more superficial pectoral fascia.

Tebbetts [8] reviewed the potential disadvantages of the subfascial pocket location. It is extremely thin to cover the implant. In my experience involving more than 3,000 cases, this dissection is still a good choice, particularly for patients with thick breast tissue.

Only a low incidence of bottom out (the descent of the implant) was observed in our study. The reason being that a good pocket dissection in the lower pole with the endoscope and a sharp dissection created a surgical pocket. This dissection cannot be performed bluntly without an endoscope. The descent of the large implant may be due to the weakness of the muscle in the fascial tissue at the new inframammary fold. The dissection below the seventh and eighth intercostal rib is generally a weak fascial point of rectus abdominis and external oblique muscle [6].

In 35 patients that received a smooth gel insertion, a high incidence of capsular contracture was observed compared to that of the textured round gel group. The inclusion criteria for this group were: multiple pregnancies and breast ptosis. Based on the criteria, it is believed that such high incidences of smooth gel in the subfascial plane may be due to the limited movement of the pectoralis major muscle. Capsular contracture of smooth gel implant in submuscular plane is less than $5 \%$ which has been observed by the author of this study, based on the author's 30-years of experience involving 6,932 cases from 1987 to 2007.

In the textured anatomical implant group, incidences of capsular contracture, rippling, implant rotation and implant border palpation were exceptionally high. The reason for higher incidence of capsular contracture in the textured anatomical implant compared to the textured round implant is not clear.

The integration of the fascia after subfascial breast augmentation without an endoscope was studied in detail [9]. It was found that the $45.0 \%$ of the fascia were broken and split. An endoscope and other special instruments are required to ensure the accuracy of the technique, achieve a perfect dissection plane and create an optimal pocket. Bleeding can be checked and it is possible to ensure cauterization as well. Rechecking the bleeding point after the implant insertion is also possible as a final step in preventing hematoma. 
The incision length in our series is not short, mainly because of the difficulty of textured gel round implant insertion used mostly in our operation. Capsular contracture rate is exceptionally high in patients that receive smooth gel implants. The incision length is likely shorter in patients with smooth gel insertions.

In conclusion, subfascial endoscopic transaxillary breast augmentation can be safely used in patients with thick breast tissue to achieve excellent results, both for good cleavage and good breast shape. Rippling and implant border palpation may occur frequently in patients with thin breast tissue. So careful selection of the technique and patient is the key. The capsular contracture with round textured implant is very low, but exceptionally high in the smooth gel implant group and the textured anatomical group. The high incidence of capsular contracture, rippling, implant border palpation, and implant rotation was observed specifically in the textured anatomical implant group that required revision surgery.

\section{Conflicts of interest}

The author has nothing to disclose.

\section{References}

1. Benito-Ruiz J. Nipple shields in transaxillary breast augmen- tation. Ann Plast Surg 2017;78:397-402.

2. Handel N. Transumbilical breast augmentation. Clin Plast Surg 2009;36:63-74, vi.

3. Tebbetts JB. Incision approaches. In: Tebbetts JB, editor. Augmentation mammaplasty. Edinburgh: Mosby, Elsevier; 2010. p. 219-30.

4. Graf RM, Bernardes A, Auersvald A, Damasio RC. Subfascial endoscopic transaxillary augmentation mammaplasty. Aesthetic Plast Surg 2000;24:216-20.

5. Stoff-Khalili MA, Scholze R, Morgan WR, Metcalf JD. Subfascial periareolar augmentation mammaplasty. Plast Reconstr Surg 2004;114:1280-8; discussion 1289-91.

6. Aygit AC, Basaran K, Mercan ES. Transaxillary totally subfascial breast augmentation with anatomical breast implants: review of 27 cases. Plast Reconstr Surg 2013;131:1149-56.

7. Salgarello M, Visconti G, Barone-Adesi L. Nipple-sparing mastectomy with immediate implant reconstruction: cosmetic outcomes and technical refinements. Plast Reconstr Surg 2010;126:1460-71.

8. Tebbetts JB. Implant pocket locations. In: Tebbetts JB, editor. Augmentation mammaplasty. Edinburgh: Mosby, Elsevier; 2010. p. 181-217.

9. Jinde L, Xiaoping C, Wanquan Z, Xia G, Ligang X. Can the pectoral fascia integrity be preserved during subfascial breast augmentation through the axillary approach? Aesthetic Plast Surg 2010;34:29-32. 